\title{
GGE Biplot Analysis for Yield Stability in Multi-environment Trials of Promising Hybrid Rice (Oryza sativa L.)
}

\author{
Anowara Akter ${ }^{1}$, M Jamil Hasan¹, Umma Kulsum¹, M H Rahman¹, M Khatun ${ }^{2}$ and M \\ R Islam²
}

\begin{abstract}
The genotype and genotype by environment biplot model is an excellent tool for visual multienvironment trials data analysis. In this study we investigated grain yield of six rice genotypes (three tested, one released hybrids and two inbred check varieties) in five environments. The combined analysis of variance for grain yield data indicated that the differences among all sources of variation were highly significant $(\mathrm{P}<0.001)$. Environment $(\mathrm{E})$, Genotype $(\mathrm{G})$ and $\mathrm{G} \times \mathrm{E}$ interaction effects accounted for $12.49,76.51$ and $10.21 \%$ of the total sum of squares respectively. The first two principal components (PC1 and PC2) were used to display a two-dimensional GGE biplot. Thus, genotypic PC1 scores $>0$ classified the high yielding genotypes while PC1 scores $<0$ identified low yielding genotypes. Unlike genotypic PC1, genotypic PC2 scores discriminated the unstable ones. The GGE biplot analysis was useful in identifying stable genotypes with high yield performance. In this study, the polygon view of GGE biplot showed that the vertex genotypes were BRRI1A/BR168R (G1), BRRI10A/BRRI10R (G2) and BRRI dhan28 (G5) having the largest distance from the origin, which was most discriminated genotypes with the unstable ones. These vertex genotypes BRRI1A/BR168R (G1) and BRRI10A/BRRI10R (G2) gave higher yield (PC1 scores $>0$ ) while another vertex genotype BRRI dhan28 (G5) produced low yield (PC1 score $<0$ ). Hence, the vertex genotype BRRI10A/BRRI10R (G2) was high yielding for all environments and it fell into section 1 following IR58025A/BRRI10R (G3) and BRRI hybrid dhan1 (G4). Mean yield and stability performance over environments of each genotype is explored by using the average environment (tester) coordinate (AEC) methods. These methods show that the genotypes BRRI10A/BRRI10R (G2), IR58025A/BRRI10R (G3) and BRRI hybrid dhan1 (G4) had higher stability as well as higher mean yield while the genotype IR58025A/BRRI10R (G3) had the highest stability out of these three genotypes. The ideal genotype biplot suggests that the closer to 'ideal' genotype was IR58025A/BRRI10R (G3) followed by G2 and G4 being more desirable than the other genotypes. Similarly, the environment Barisal (E3) was 'ideal' environment followed by E1 (Gazipur), E2 (Comilla) and E5 (Satkhira). Hence, the environment Barisal (E3) is more stable and suitable for all genotypes following Satkhira (E5) because it has large PC1 and small PC2 score but Rangpur (E4) is a discriminating environment because it has large PC2 score. The interrelationship among the environments according to the small angles of test environments was highly positively correlated. Gazipur (E1), Comilla (E2), Barisal (E3) and Satkhira (E5) were closely correlated with small angles but Rangpur (E4) had medium long angles. Comparison between two genotypes showed that BRRI10A/BRRI10R (G2) and IR58025A/BRRI10R (G3) were high yielder in test environments. Thus, the difference between G2 and G3 was relatively small in test environments.
\end{abstract}

Keywords: GGE biplot analysis, yield stability, multi-environments, hybrid rice

\section{INTRODUCTION}

Plant breeders perform multienvironment trials (MET) to evaluate new improved genotypes across test environments (several locations), before a specific genotype is released for production to supply growers. In such experiments, genotype-environment (GE) interaction is a commonly evaluated (Annicchiarico, 2002; Kang, 1998; Karimizadeh et al., 2012a; Yan et al., 2007). Genotype × environment (GE) interaction refers to different ranking of genotypes across environments and may complement the selection process and recommendation of a genotype for a target environment (Ebdon and Gauch, 2002; Gauch, 2006).

${ }^{1}$ Hybrid Rice Division, and 2Plant Breeding Division, Bangladesh Rice Research Institute, Gazipur 1701, Bangladesh. *Corresponding author's E-mail: anowaraa@yahoo.com 
It may also reduce the selection efficiency in different breeding programs because in a GE interaction, measured traits are less predictable and cannot be interpreted using main effects (genotype or environment) and need more analysis (Gauch et al., 2008). GE interaction is also one of the most important reasons for the failure or decreased efficiency of breeding efforts to serve small resource poor farmers in different areas (Ceccarelli, 1996; Kaya et al., 2006 and Mitrovic et al., 2012). Plant breeders perform multi-environment trials (MET) to select favourable genotypes based on both mean yield and performance stability and to determine whether a test environment is homogeneous should be divided into various mega-environments (Gauch, 2006; Yan and Kang, 2003). Different statistical model were used to describe GE interaction and facilitate genotype recommendations in MET such as stability variance (Shukla, 1972), coefficient of variability (Francis and Kanneberg, 1978) and additive main effects multiplicative interaction (AMMI) (Gauch and Zobel, 1988; Zobel et al., 1988; Gauch, 2006) have been commonly used to analyze MET data to reveal patterns of GE interaction. Yan et al. (2000) proposed another methodology known as genotype and genotype by environment (GGE) biplot for graphical display of GE interaction pattern of MET data with many advantages. GGE biplot analysis considers both genotype (G) and GE interaction effects and graphically displays GE interaction in a two way table (Yan et al., 2001). GGE biplot is an effective method based on principal component analysis (PCA) to fully explore MET data. It allows visual examination of the relationships among the test environments, genotypes and the GE interactions. The main objectives of the present study are to identify the best performing high yielding stable promising hybrids for selection environments, the identification of megaenvironments and analysis of the ideal genotype and environment by GGE biplot method.

\section{MATERIALS AND METHODS}

Experimental design and plant materials. The experiments were conducted at five districts namely Gazipur (E1), Comilla (E2), Barisal (E3), Rangpur (E4) and Satkhira (E5) representing five different agroecological zones (AEZ) of Bangladesh during Boro season 2007-08. Six genotypes consisting of three advanced lines (BRRI1A/BRRI168R (G1), BRRI10A/BRRI10R (G2) and IR58025A/BRRI10R (G3), one released hybrid (BRRI hybrid dhan1 (G4) and two inbreed check varieties (BRRI dhan28 (G5) and BRRI dhan29 (G6) were used as experimental materials. The experiment was laid out in a randomized complete block (RCB) design with three replications. Thirty-day-old seedlings were transplanted in 20 square meter plot using single seedling per hill spacing at $20 \mathrm{~cm} \times 15 \mathrm{~cm}$. Fertilizers were applied @ 270-130-1200-70-10 kg/ha urea, TSP, MP, gypsum and $\mathrm{ZnSO}_{4}$ respectively. Standard agronomic practices were followed and plant protection measures were taken as required following the recommendation of Adhunik Dhaner Chash, BRRI (2008). Two border rows were used to minimize the border effects. The grain yield (tha-1) data were collected at $14 \%$ moisture level. Data were collected followed by standard method as described by Yoshida et al. (1976).

Statistical analysis. The grain yield data for six genotypes in five environments were used to combined analysis of variance (ANOVA) to determine the effects of environment $(\mathrm{E})$, genotype $(\mathrm{G})$ 
and their interactions. The data were graphically analyzed for interpreting GE interaction using the GGE biplot software (Yan, 2001). GGE biplot methodology, which is composed of two concepts, the biplot concept (Gabriel, 1971) and the GGE concept (Yan et al., 2000), was used to visually analyze the rice genotypes MET data. This methodology uses a biplot to show the factors ( $G$ and GE) that are important in genotype evaluation and that are also the sources of variation in GE interaction analysis of MET data (Yan, 2001). The graphs were generated based on (i) The polygon view of GGE biplot to identification of winning genotypes and their mega environments by 'which-won-where' pattern, Ranking of genotypes based on yield and stability performance, (iii) Evaluation of genotypes related to an ideal genotypes, (iv) Evaluation of environments related to ideal environments, (v) Relationship among environments and (vi) Comparison between two genotypes.

\section{RESULTS AND DISCUSSION}

Combined analysis of variance. Table 1 presents the combined analysis of variance for grain yield of six rice genotypes. Genotype (G), environment (E) and genotype $\times$ environment interaction (GEI) were highly significant $(\mathrm{P}<0.001)$ for grain yield. The highly significant $G \times E$ effects suggest that genotypes may be selected for adaptation to specific environments, which is in harmony with the findings of Aina et al. (2009) and Xu Fei-fei et al. (2014) in G × E interaction effects of cassava genotypes. Hence, the genotype $\times$ environment interaction effects demonstrated that genotypes responded differently to the variation in environmental conditions of locations, which indicated the necessity of testing rice varieties at multiple locations. This also shows the difficulties encountered by breeders in selecting new varieties for release. The factors explained (\%) showed that rice grain yield was affected by genotype (76.51\%), environment $(12.49 \%)$ and their interaction $(10.21 \%)$.

Table 1. Combined analysis of variance of grain yield for six rice genotypes evaluated at five environments.

\begin{tabular}{lllll}
\hline Source & $\mathrm{df}$ & $\mathrm{SS}$ & MS & Explained SS $(\%)$ \\
\hline Genotype $(\mathrm{G})$ & 5 & 65.444 & $13.089^{* * *}$ & 76.51 \\
Environment (E) & 4 & 10.683 & $2.671^{* * *}$ & 12.49 \\
G $\times$ E interaction (GEI) & 20 & 8.739 & $0.437^{* * *}$ & 10.21 \\
Error & 60 & 0.669 & 0.011 & \\
Total & 89 & 85.535 & 0.961 & \\
\hline
\end{tabular}

***Indicate significance at $\mathrm{P}<0.001$ probability level; $\mathrm{df}=$ degree of freedom; $\mathrm{SS}=\mathrm{Sum}$ of square; MS=Mean of square.

Polygon view of GGE biplot analysis of MET data. The polygon view of GGE biplot (Fig. 1) is the best way for the identification of winning genotypes with visualizing the interaction patterns between genotypes and environments (Yan and Kang, 2003) in MET data analysis, which is helpful in estimating the possible existence of different mega environments (Gauch and Zobel, 1997; Yan and Rajcan, 2002; Yan and Tinker, 2006). In this biplot, a polygon was formed by connecting the vertex genotypes with straight lines and the rest of the genotypes were placed within the polygon. The vertex genotypes were G1, G2 and G5 having the largest distance from the origin. These genotypes are the best or poorest in some or all environments because they are farthest from the origin of biplot (Yan and Kang, 2003), which were more responsive to environmental change and are considered as specially adapted genotypes. There are three rays in Figure 1 , which divided the biplot into three 
sections. The genotypes fell into three sections but all the tested environments fell into section 1 .

The first section contains three genotypes G2, G3 and G4 and the vertex genotype for this section was G2, suggesting the high yielding genotype for these five locations following G3 and G4. The second section contains two genotypes G5 (vertex genotypes) and G6 were poorest yielding genotypes in any environments. The other vertex genotype G1 gave medium high yield which fell in section 3; this genotype is poorly adapted to five of the testing environments. On the other hand, the genotype, which was located near the origin, was less responsive than the corner (vertex) genotypes. Hence, the G3 and G6 were located apparently near the biplot origin showed moderately average performance and these genotypes were less responsive to environments than the vertex genotypes. According to the findings of Yan and Tinker (2006), the vertex genotypes were the most responsive genotypes, as they have the longest distance from the origin in their direction. In the present investigation, the partioning of GE interaction through GGE biplot analysis showed that PC1 and PC2 accounted for $84.7 \%$ and $9.9 \%$ of GGE sum of squares, respectively, explained $94.6 \%$ of the total variance (Fig. $1)$.

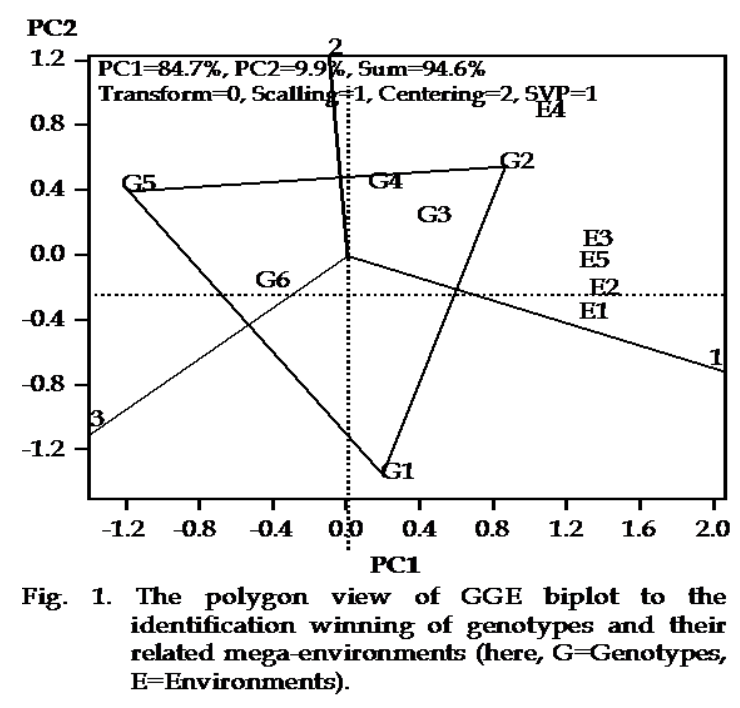

4 Akter et al
Ranking of genotypes based on mean yield and stability performance. In GGE biplot methodology, the estimation of yield and stability of genotypes (Fig. 2) were done by using the average environment (tester) coordinate (AEC) methods (Yan, 2001; Yan and Hunt, 2001). The line passing through the biplot origin is called the average environment (tester) coordinate (AEC), which is defined by the average PC1 and PC2 scores for all environments (Yan and Kang, 2003). More close to concentric circle indicates higher mean yield. The line, which passes through the origin and is perpendicular to the AEC with double arrows, represents the stability of genotypes. Either direction away from the biplot origin, on the axis, indicates greater GE interaction and reduced stability. For selection, the ideal genotypes are those with both high mean yield and high stability. In the biplot, they are close to the origin and have the shorter vector from the AEC. Thus, genotype G1 was the least stable and genotypes G2, G3, G4 and G6 were the most stable. On the other hand, the genotypes on the right side of the line with double arrows have yield performance greater than mean yield and the genotypes on the left side of this line had yields less than mean yield. In this study, the genotypes G2, G3 and G4 had the higher stability as well as higher mean yield and eventually had been constellated into the same group. However, G3 had the highest stability out of these three genotypes. These results are in agreement with those obtained by Nahief (2013) in wheat. 


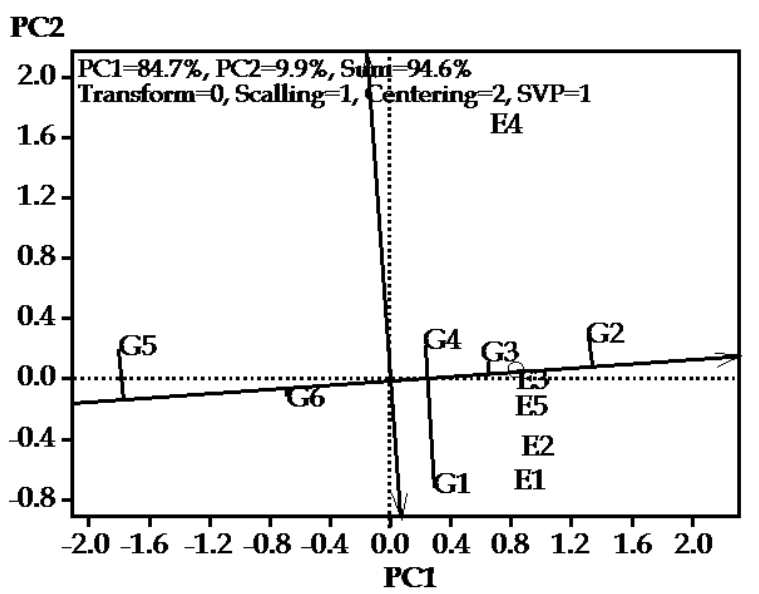

Fig. 2 GGE biplot showing the ranking of genotypes for both yield and stability performance over environments.

Evaluation of genotypes relative to ideal genotypes. An interesting application of GGE biplot software is the evaluation of genotype relative to an ideal genotype. The ideal genotype as virtual genotype is one that has both high mean yield across test environments and is absolutely stable in performance (Yan and Rajcan, 2002; Yan and Kang, 2003; Farshadfar et al., 2012). This genotype has large PC1 scores (high mean yield) and small (absolute) PC2 scores (high stability). Although such an 'ideal' genotype may not exist in reality, it could be used as a reference for genotype evaluation (Mitrovic et al., 2012). A genotype is more desirable if it is closer to 'ideal' genotype (Kaya et al., 2006 and Mitrovic et al., 2012). Therefore, G3 was closer to the 'ideal' genotype followed by G2 and G4 being more desirable than other genotypes (Fig. 3). On the other hand, the low yielding genotypes (G5 and G6) were considered to be undesirable because they are placed far from the ideal genotypes. It seems that identification of ideal genotype through GGE biplot methodology is a proper tool for identifying most stable high yielding genotypes. This method can be regarded as same as AMMI parameter, which allow to facilitate identifying more stable genotypes using AMMI procedure (Sabaghnia et al., 2008a). In other words, identification of ideal genotype procedure attempts to define the GE interactions by one parameter (distance from ideal genotype) and summarize complex aspect of GE interaction using only one parameter.

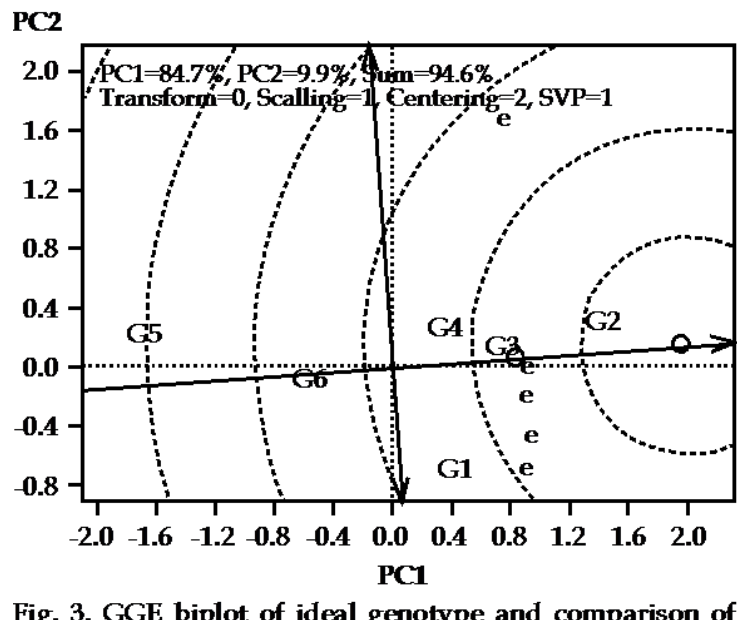

Fig. 3. GGE biplot of ideal genotype and comparison of the genotypes with respect to the ideal genotype.

Evaluation of environments relative to ideal environments. According to Yan (2001), discriminating ability and representativeness are the important properties of a test location. An ideal location should be highly differentiating for the tested genotypes and at the same time representative of the target location (Yan and Kang, 2003). Similar to ideal genotype, an ideal environment or location is defined and showed by the small circle with an arrow pointing to it. Figure 4 shows the environment E3 as an 'ideal' environment. The ranking of other environments based on the ideal nature of environments were E5>E2>E1 (Fig. 4). The environment E3 has large PC1 score and small PC2 score. Hence, this environment is more stable and suitable for all genotypes following E5. On the other hand, E4 is a discriminating environment because it has large PC2 score. The discriminating ability of a location is concerned with the composition of genotypes, but the presence of GE interaction complicates the identification of an ideal test location 
(Yan et al., 2000). GGE methodology is interactions partioning them into their PCs. The test environments should have large PC1 scores in order to discriminate genotypes in terms of the genotypic main effect and absolute small PC2 scores in order to be more representative of the overall locations (Yan and Rajcan, 2002).

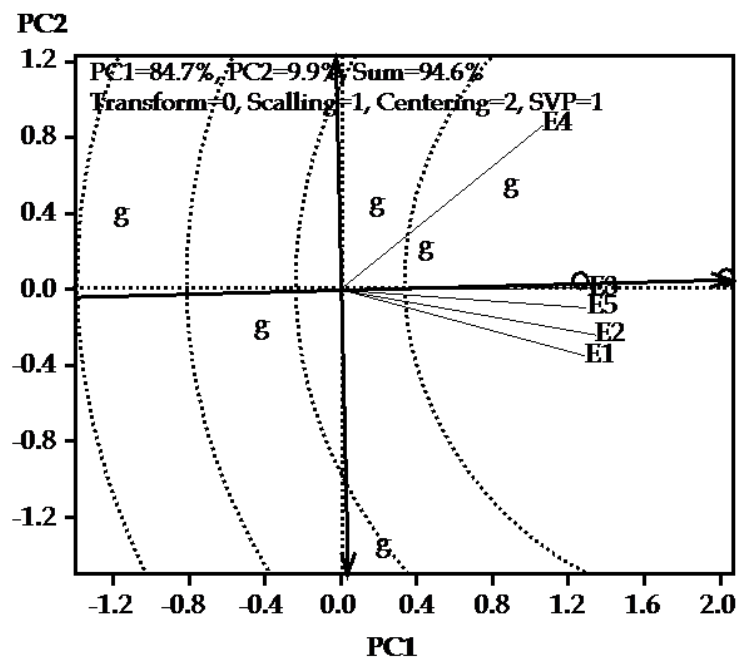

Fig. 4. GGE biplot of ideal environment and comparison of the environments with the ideal environment.

Relationship among environments. The GGE biplot showed in Figure 5 explained $94.6 \%$ of the total variation and so this biplot can be used for extracting interrelationships among the environments. The environment vectors are lines that connect the biplot origin and the markers of test environments and the angle between them is related to the correlation coefficient (Kroonenberg, 1995). On the other hand, the biplot vector view is mainly used to identify test environments, which have large angle or low or negative correlations. Locations with small angles between them were highly positively correlated and they provided similar information on genotypes. According to the angles of test location vectors, the five locations are grouped into two major groups. One group includes E1, E2, E3 and E5 was closely correlated (Fig. 5), suggesting that the locations provide redundant information about genotypes while the other group involves E4. Obtaining suitable tool to analyze this kind of similar information by using fewer test environments generally reduces the cost of testing and increases breeding efficiency. In addition, in the vector view of the biplot, the length of the location vectors estimates the standard deviation within each location, which is a measure of their discriminating ability.

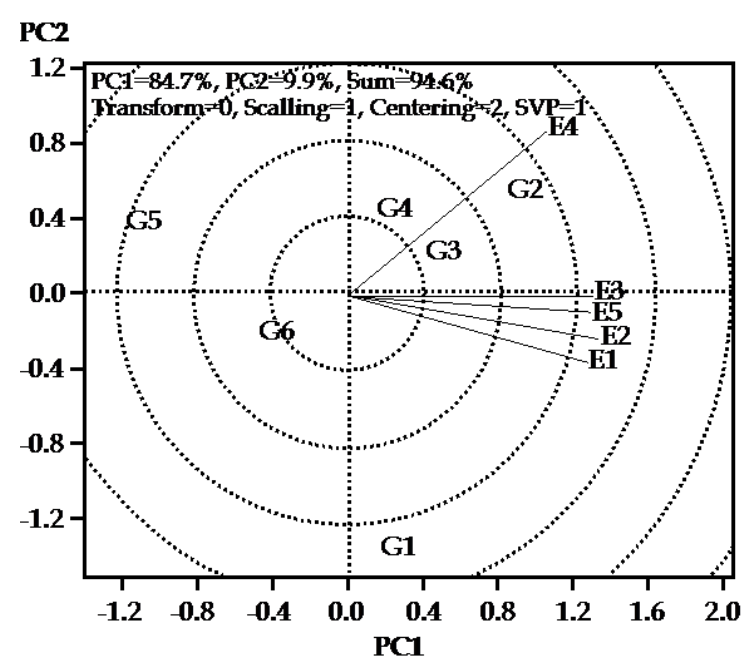

Fig. 5. GGE biplot for relationships among test environments.

Comparison between two genotypes. In a GGE biplot, two genotypes can be visually compared by connecting them with a straight line followed by drawing a perpendicular line that passes through the biplot origin (Fig. 6). This perpendicular line is the 'equality line' of the two genotypes. That is, the two genotypes to be compared should be equal in all environments that are located on this line. Since the biplot distance of the line that connects the two genotypes measures the Euclidian distance between them, comparison using the method shown in Figure 6 is meaningful, because the connection line is not so long. Genotype has higher values in environments that are located on its side of the equality line. The genotypes, BRRI10A/BRRI10R (G2) and IR58025A/BRRI10R (G3) had higher yield in all environments. Thus, the difference between G2 and G3 was relatively small in all environments. 


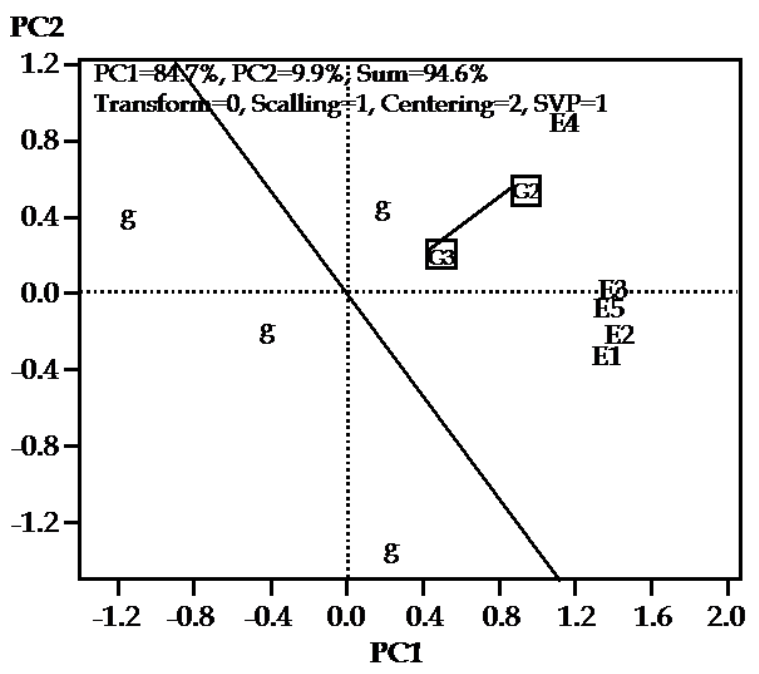

Fig. 6. GGE biplot for comparison between two best genotypes with probability of difference in $\mathbf{G}: \mathbf{P}$ $<=0.0984$.

\section{CONCLUSIONS}

The GGE biplot model is an excellent tool for visual MET data analysis (Mohammadi et al., 2011). In this study, the combined analysis of variance (ANOVA) indicates that the genotype (G), environments (E) and $G \times E$ interaction variance were highly significant at $\mathrm{P}<0.001$. On the other hand, the vertex genotype G2 was high yielding genotype for all environments and this genotype fell into section 1 following G3 and G4. Other two vertex genotypes G1and G5 gave medium high yield and poor yield with poor adaptation to five testing environments.

Mean yield and stability performance over environments of each genotype is explored by using the average environment (tester) coordinate (AEC) methods. These methods show that the genotypes G2, G3 and G4 had higher stability as well as higher mean yield while the genotype G3 had the highest stability out of these three genotypes. The ideal genotype biplot suggests that the closer to 'ideal' genotype was G3 followed by G2 and G4 being more desirable than other genotypes. Similarly, the environment E3 was 'ideal' environment followed by E1, E2 and E5.
Hence, the environment E3 is more stable and suitable for all genotypes following to E5 because it has large PC1 and small PC2 score but E4 is a discriminating environment because it has large PC2 score. The interrelationship among the environments according to the small angles of test environments between them were highly positively correlated such as E1, E2, E3 and E5 were closely correlated with small angles but E4 had medium long angles. Comparison between two genotypes showed that G2 and G3 had higher yield in test environments. Thus, the difference between G2 and G3 was relatively small in test environments.

\section{ACKNOWLEDGEMENTS}

Authors are thankful to all the staffs of Hybrid Rice Research and Development GOB project funded by Ministry of Agriculture and the yield trials conducted in different locations was supported by late Dr A W Julfiquare, Director (Admin), Project Director of Hybrid Rice Research and Development Project, Bangladesh Rice Research Institute, Gazipur 1701, Bangladesh and also express thanks to $M \mathrm{R}$ Islam for helping GGE biplot statistical analysis.

\section{REFFERENCES}

Annicchiarico, P. 2002. Defining adaptation strategies and yield stability targets in breeding programmes, 365-383 p. In: Kang MS (Ed.). Quantitative genetics, genomics, and plant breeding, Wallingford, UK, CABI.

Aina, O O, A G O Dixon, llona Paul and E A Akinrinde. 2009. G $\times E$ interaction effects on yield and yield components of cassava (landraces and improved) genotypes in the savanna regions of Nigeria. African Journal of Biotechnology, 8(19), pp. 4933-4945.

BRRI. 2008. Adhunik Dhaner Chash, 15th edition. Bangladesh Rice Research Institute, Gazipur 1701, Bangladesh. p. 20-50.

Ceccarelli, S. 1996. Adaptation to low or high input cultivation. Euphytica, 92: 203-214. 
Ebdon, J S and H G Gauch. 2002. Additive main effect and multiplicative interaction analysis of national turfgrass performance trials: I. Interpretation of genotype $\times$ environment interaction. Crop Sci. 42: 489-496.

Farshadfar, E, R Mohammadi, M Aghaee, Z Vaisi. 2012. GGE biplot analysis of genotype $x$ environment interaction in wheat-barley disomic addition lines. Aust. J. Crop Sci. 6(6): 1047-1079.

Francis, T R and L W Kannenberg. 1978. Yield stability studies in short season maize. I. A descriptive method for grouping genotypes. Can J Plant Sci. 58: 1029-1034.

Gabriel, K R. 1971. The biplot graphic display of matrices with application to principal component analysis. Biometrica, 58: 453-467.

Gauch, H G, R W Zobel. 1988. Predictive and postdictive success of statistical analysis of yield trials. Theor. Appl. Gent. 76-10.

Gauch, H G and R W Zobel. 1997. Identifying mega-environments and targeting genotypes. Crop Sci. 37: 311-326.

Gauch, H G. 2006. Statistical analysis of yield trials by AMMI and GGE. Crop Sci. 46: 14881500.

Gauch, H G, H P Piepho and P Annicchiaricoc. 2008. Statistical analysis of yield trials by AMMI and GGE. Further considerations. Crop Sci. 48: 866-889.

Kaya, Y, M Akcura and S Taner. 2006. GGEbiplot analysis of multi-environment yield trials in bread wheat. Turk. J. Agric. 30: 325337.

Kang, M S. 1998. Using genotype-by-environment interaction for crop cultivar development. Adv Agron 62: 199-252.

Karimizadeh, R, M Mohammadi, N Sabaghnia and M K Shefazadeh. 2012a. Using Huehn's Nonparametric Stability Statistics to investigate Genotype $\times$ Environment Interaction. Not Bot Horti Agrob. 40: 195-200.

Kroonenberg, P M. 1995. Introduction to biplots for $G \times E$ Tables. Dep. Of Mathematics, Res. Rep. 51. Univ. of Queensland, Australia.

Mitrovic, B, D Stanisavljevi, S Treski, M Stojakovic, M Ivanovic, G Bekabac and M Rajkovic. 2012. Evaluation of experimental maize hybrids tested in multi-location trials using AMMI and GGE biplot analysis. Turkish J. Field Crops. 17(1): 35-40

Mohammadi, R, M Armion, D Sadeghzadeh, A Amri and M Nachit. 2011. Analysis of genotype- by-environment interaction for agronomic traits of durum wheat in Iran. Plant Prod Sci. 14: 15-21.

Naheif, E, M Mohammad. 2013. Genotype by environment interactions for grain yield in bread wheat (Triticum aestivum L.). Global Sci. Res. J. 1(1): 045-052.

Sabaghnia, N, H Dehghani and S H Sabaghpour. 2008a. Graphic analysis of genotype $\times$ environment interaction of lentil yield in Iran. Agron. J. 100: 760-764.

Shukla, G K. 1972. Some statistical aspects of portioning genotype-environmental components of variability. Heridity 29: 237245.

$\mathrm{Xu}$, Fei-fei, TANG Fu-fu, SHAO Ya-fang, CHEN Ya-ling, TONG chuan and BAO Jing-song. 2014. Genotype $\times$ Environment Interaction for Agronomic Traits of Rice Revealed by Association Mapping. Rice Sci. 21(3): 133-141.

Yan, W, L A Hunt, Q Sheng and Z Szlavnics. 2000. Cultivar evaluation and mega environment investigation based on the GGE biplot. Crop Sci. 40: 597-605.

Yan, W. 2001. GGE biplot a windows application for graphical analysis of multi-environment trial data and other types of two way data. Agron. J. 93: 1111-1118.

Yan, W and L A Hunt. 2001. Interpretation of genotype $\times$ environment interaction for winter wheat yield in Ontario. Crop Sci 41: 19-25.

Yan, W and I Rajcan. 2002. Biplot analysis of test sites and trait relations of soyabean in Ontario. Crop Sci. 42: 11-20.

Yan, W and M S Kang. 2003. GGE biplot analysis: a graphical tool for breeders, Geneticists and Agronomists. 1st Edn., CRC Press LLC., Boca Raton, Florida, pp: 271.

Yan, W and N A Tinker. 2006. Biplot analysis of multi-environment trial data: Principles and applications. Can J Plant Sci. 86: 623-645.

Yan, W, M S Kang, B Ma, S Woods and P L Cornelius. 2007. GGE biplot vs. AMMI analysis of genotype- by-environment data. Crop Sci. 47: 643-655.

Yoshida, S, D A Forno, J H Cock and K A Gomez. 1976. Laboratory manual for physiological studies of rice. (3rd edn), International Rice Research Institute, Los Banos, Philippines.

Zobel, R W, M J Wright, H G Gauch. 1988. Statistical analysis of a yield trial. Agron. J. 80: 388-393. 